\title{
ON Comparison, Equivalence AND AdDition of MAGNITUDES
}

\author{
Paulo A. Veloso \\ Federal University of Rio de Janeiro. \\ National Council for Scientific and Technological Development (CNPq), BRASIL \\ pasveloso@gmail.com \\ ABel LASSALLe-CASANAVE \\ Federal University of Bahia. \\ National Council for Scientific and Technological Development (CNPq), BRASIL \\ abel.lassalle@gmail.com \\ EDUARDO N. GIOVANNINI \\ Universidad Nacional del Litoral. \\ National Scientific and Technical Research Council (CONICET), ARGENTINA \\ engiovannini@conicet.gov.ar
}

\begin{abstract}
A theory of magnitudes involves criteria for their comparison, equivalence and addition. We examine these aspects from an abstract viewpoint, stressing independence and definability. These considerations are triggered by the so-called De Zolt's principle in the theory of equivalence of plane polygons.
\end{abstract}

Keywords: Theory of magnitudes $\bullet$ comparison $\bullet$ equivalence $\bullet$ addition $\bullet$ independence $\bullet$ definability $\bullet$ geometry $\bullet$ De Zolt's principle

\section{Introduction}

A usual procedure in the development of the concept of magnitude consists in defining magnitude as an ordered commutative or Abelian semigroup. Historically, this conception has its roots in the first modern axiomatizations of the notion of magnitude, in the works of Otto Stolz (1883, 1885), Otto Hölder (1901) and Edward Huntington (1902). A main motivation in the emergence of the modern theory of magnitude was to provide a general axiomatic foundation for this notion, which could adequately incorporate "numerical" as well as "geometrical" kinds of magnitudes. Metatheoretical issues, such as the independence of continuity conditions (viz., the Archimedean axiom) from other axioms of magnitude, were also a central concern in this context. Philosophically, the main goal was to achieve a conceptual elucidation of perhaps the most fundamental mathematical concept of Greek mathematics, but which was never explicit or clearly explained. 
The "standard" analysis of the concept of magnitude can be described in schematic terms as follows. ${ }^{1}$ One start by postulating a relation of equivalence $\sim$ for a given set of elements which satisfies the usual following properties:

$(\operatorname{Rf}[\sim])$ Reflexive

$(\operatorname{Sm}[\sim])$ Symmetric

( $\operatorname{Tr}[\sim])$ Transitive for every a: a $\sim$ a.

for all $a, b:$ if $a \sim b$, then $b \sim a$.

Then, one introduces a binary operation of addition (;) which satisfies the associative property:

(Asc) Associativity

for all $a, b, c:(a ; b) ; c=a ;(b ; c)$.

We thus obtain the structure of a semigroup. If the addition operation also satisfies the commutative property, the semigroup is called commutative or Abelian:

(Com) Commutativity

for all $a, b: a ; b=b ; a$.

A relation of strict ordering can be then introduced with the help of the operation of addition, by postulating that this operation also satisfies the comparability property:

(Cmp) Comparability

for all a, b, there exists a c such that exactly one of the following conditions holds:

$$
a=b ; c \quad \text { or } \quad b=a ; c \quad \text { or } \quad a=b
$$

The latter condition suggests the following definition of strict order:

$(\prec)$ Strict order $\quad a \prec b \quad$ iff $\quad$ there exists a $c$ such that $a$; $c=b$.

One can employ the properties of equivalence and addition specified above to prove that this relation of ordering satisfies the following properties:

$(\operatorname{Tr}[\prec]) \quad$ Transitive for all $a, b, c$ : if $a \prec b$ and $b \prec c$, then $a \prec c$.

$($ Asm $[\prec]) \quad$ Asymmetric for all $a, b$ : if $a \prec b$ then $b \nprec a$

and if $b \prec a$ then $a \nmid b$

(Tri $[\prec])$ Trichotomy for all $a, b$ :
$a \prec b$
or
$b \prec a$
or $a=b$. 
The relation of order thus defined is then a strict total ordering. ${ }^{2}$ Finally, one further assume that the addition also satisfies the monotonicity law, namely:

(Mn) Monotonicity

$$
\text { for all } a, b, c \text { : if } a \prec b \text {, then } a \text {; } c \prec b \text {; } \text {. }^{3}
$$

A related analysis of the concept of magnitude, but with a very different goal, was offered by Łomnicki (1922). ${ }^{4}$ In particular, Łomnicki develops a system of axioms for magnitudes, with three primitive concepts $(\sim,<,>)$, with the aim of investigating the interpretation of these axioms within the geometrical theory of equivalence. ${ }^{5}$ Another central concern here was to achieve a better understanding of the logical relations between the general axioms of order of magnitudes and the fundamental proposition about polygonal area known as De Zolt's principle. This principle is usually stated as follows: "If a polygon is divided into polygonal parts in a given way, then the union of all but one of these parts is not equivalent to the given polygon". ${ }^{6}$ The central significance of this principle for the theory of geometrical equivalence concerned the existence of a relation of (total) order for plane polygons: if this proposition would not be valid, a polygon could be lesser in area than itself, and (geometrical) equivalence and non-equivalence could not be regarded as magnitude relations.

Łomnicki postulates as axioms the above specified properties of equivalence (reflexive, symmetric and transitive) and three properties of strict order, namely transitive, trichotomy and the following condition:

(Sco[ $\succ])$ Strict converse order

for all $a, b$ : if $a \succ b$ then $b \prec a$.

An interesting problem originally investigated by Łomnicki concerned the formulation of trichotomy. Łomnicki claimed that in the presence of the axioms of equivalence $(\sim)$ the trichotomy axiom can be obtained by means of two logical independent propositions. He designated these propositions the completeness of trichotomy and the principle of disjunction. More specifically, Łomnicki suggested that the latter proposition could be conceived as an "abstract version" of De Zolt's principle, within the framework of a general theory of magnitudes. These propositions are then stated as follows:

$(\operatorname{Ctr}[\prec]) \quad$ Completeness of trichotomy

for all $a, b$ : if $a \not b$, then either $a \succ b$ or $a \prec b$.

$(\mathrm{Dzt}[\prec]) \quad$ De Zolt for all $a, b:$ if $a \sim b$, then $a \nmid b$.

Łomnicki's concise analysis into the concept of magnitude suggests us that a challenging issue for the development of a theory of magnitudes consists in incorporating or reflecting the specific geometrical properties stated in De Zolt's principle. In other 
words, a starting point for the construction of a "well-behaved" theory of magnitude might consist not only in formulating the latter principle in an adequate and precise way, but also in deriving it from the basic principles of equivalence, comparison and addition of magnitudes. The analysis of the concept of magnitude presented in the next sections is then oriented towards the problem of providing a proof of De Zolt's principle, in an abstract setting. Although this proof will be provided in another work, the present examination will be relevant for the understanding of the logical relations between the properties of comparison, equivalence and addition of magnitudes. Section 2 examines principles and properties of comparison and equivalence of magnitudes. Section 3 considers magnitude addition and comparison. Section 4 presents some concluding remarks. Appendix A fills in the details of the mathematical results presented here.

\section{Comparison and Equivalence}

We begin with basic and derived properties of comparison and equivalence. We also raise some questions regarding independence and definability. As basic properties of equivalence we take the already specified properties of identity $=$ : reflexive $(\operatorname{Rf}[\sim])$, symmetric $(\operatorname{Sm}[\sim])$ and transitive $(\operatorname{Tr}[\sim])$. Similarly, as basic properties of comparison $\preceq$, we assume the following familiar properties of $\leq$ :

(Rf $[\preceq]) \quad$ Reflexive

( $\operatorname{Tr}[\preceq]) \quad$ Transitive

( $\mathrm{Tt}[\preceq]) \quad$ Total for every a: $a \preceq a$.

for all $a, b, c$ : if $a \preceq b$ and $b \preceq c$, then $a \preceq c$.

for all $a, b: a \preceq b$ or $b \preceq a$.

As basic properties connecting equivalence and comparison, we take some familiar properties of identity $=$ and $\leq$ :

$(\sim \Rightarrow \preceq)$ Equivalence and comparison for all $a$, $b$ : if $a \sim b$, then $a \preceq b$.

$(\preceq \Rightarrow \sim$ ) Comparison and equivalence for all $\mathrm{a}$, $\mathrm{b}$ : if $\mathrm{a} \preceq \mathrm{b}$ and $\mathrm{b} \preceq \mathrm{a}$, then $\mathrm{a} \sim \mathrm{b}$.

We can also introduce some defined comparisons.

(Ł) Converse comparison

(ঝ) Strict comparison

$(\succ)$ Strict converse comparison for all $a, b: a \succeq b$ iff $b \preceq a$. for all $a, b: a \prec b$ iff $a \preceq b$ and $a \not b$. for all $a, b: a \succ b$ iff $b \prec a$. 
We now give some models for equivalence and comparison.

Example 2.1 (Models for $\sim$ and $\preceq$ ). The basic properties of $\sim$ and $\preceq$ have numerical models with $\mathrm{a} \sim \mathrm{b}$ iff $\mathrm{a}=\mathrm{b}$ and $\mathrm{a} \preceq \mathrm{b}$ iff $\mathrm{a} \leq \mathrm{b}:(\mathbb{N})$ naturals, $(\mathbb{Z})$ integers, $(\mathbb{Q})$ rationals, $(\mathbb{R})$ reals, and their subsets consisting of the numbers above 1 .

The next result gives some derived properties of equivalence and comparison.

Lemma 2.1 (Derived properties of $\sim$ and $\preceq$ ). Equivalence $\sim$ and comparison $\preceq$ have the following derived properties.

1. For all $\mathrm{a}$, $\mathrm{b}$ : if $\mathrm{a} \sim \mathrm{b}$, then $\mathrm{b} \preceq \mathrm{a}$.

2. For all $\mathrm{a}, \mathrm{b}: \mathrm{a} \sim \mathrm{b}$ iff $\mathrm{a} \preceq \mathrm{b}$ and $\mathrm{b} \preceq \mathrm{a}$.

Proof. The first one follows from $(\sim \Rightarrow \preceq)$ by $(\operatorname{Sm}[\sim])$ and the second one follows from the first one by $(\preceq \Rightarrow \sim){ }^{7}$

We now examine questions about definability and independence. We first consider the definability of equivalence from comparison

Question 2.1. Can we define equivalence $\sim$ from comparison $\preceq$ ?

Answer. Yes: define ' $a \sim b$ ’ as “a $\preceq$ b \& b $\preceq$ a” (see Lemma 2.2; from $\preceq$.)

Lemma $2.2(\sim$ from $\preceq)$. Assume $\mathrm{a} \sim \mathrm{b} \hookrightarrow \mathrm{a} \preceq \mathrm{b} \wedge \mathrm{b} \preceq$ a. Then, $\sim$ is symmetric, $(\operatorname{Rf}[\preceq])$ yields $(\operatorname{Rf}[\sim])$ and $(\operatorname{Tr}[\preceq])$ yields $(\operatorname{Tr}[\sim])$.

Proof. $(\operatorname{Sm}[\sim])$ Clearly $\sim$ is symmetric. $(\operatorname{Rf}[\sim])$ Since a $\preceq$ a (by $(\operatorname{Rf}[\preceq])$ ), we have $\mathrm{a} \preceq \mathrm{a}$ and $\mathrm{a} \preceq \mathrm{a}$, whence $\mathrm{a} \sim \mathrm{a}$. $(\operatorname{Tr}[\sim])$ Given $\mathrm{a} \sim \mathrm{b}$ and $\mathrm{b} \sim \mathrm{c}$, we have $\mathrm{a} \preceq \mathrm{b}$, $\mathrm{b} \preceq \mathrm{a}, \mathrm{b} \preceq \mathrm{c}$ and $\mathrm{c} \preceq \mathrm{b}$, thus (by $(\operatorname{Tr}[\preceq])$ ) $\mathrm{a} \preceq \mathrm{c}$ and $\mathrm{c} \preceq \mathrm{a}$, whence $\mathrm{a} \sim \mathrm{c}$.

Proposition 2.1 (Equivalence definable). Equivalence $\sim$ is definable from comparison $\preceq$.

Proof. By Lemma 2.2 ( from $\preceq$, p. 157).

We now examine whether the totality property for comparison is independent from the other basic properties of comparison.

Question 2.2. Is the totality property for comparison $\preceq$ independent from the other basic properties for $\preceq$ ?

Answer. Yes: we have counter-models (see A.1, p. 163).

Proposition 2.2 (Totality independence). The totality property $(\mathrm{Tt}[\preceq]$ ) is independent from the basic properties $(\operatorname{Rf}[\preceq])$ and $(\operatorname{Tr}[\preceq])$. 
Proof. See A.1 (Comparison and Equivalence: details, p. 164).

We next consider the definability of comparison from equivalence.

Question 2.3. Can we define comparison $\preceq$ from equivalence $\sim$ ?

Answer. No: we have counter-models. (See A.1, p. 164.)

Proposition 2.3 ( $\preceq$ not definable from $\sim$ ). Comparison $\preceq$ is not definable from equivalence $\sim$.

Proof. See A.1 (Comparison and Equivalence: details, p. 165).

\section{Comparison and Addition: simplified approach}

We now examine comparison and simple addition. We identify some basic properties of simple addition and study independence and definability. As motivating examples of addition we consider concatenation of lines at a common point (see Example 3.1, p. 158), and gluing of polygons along a common side (see Example 3.2, p. 159). In general, one expects addition to cause an increase; however, concatenation with a single-point straight line does not alter the line (see Example 3.1, p. 158).

Example 3.1 (Concatenation of lines). Fig. 1 (p. 158) illustrates concatenation of "proper" straight lines $\mathrm{r}$ and $\mathrm{s}$ : notice that $\mathrm{r} \prec \mathrm{r} ; \mathrm{s} \succ$ s. Fig. 2 (p. 158) illustrates concatenations with single-point straight lines $\mathrm{B}$ and $\mathrm{A}$ : notice that $\mathrm{r} ; \mathrm{B}=\mathrm{r}$ and $\mathrm{A} ; \mathrm{r}=$ r.

Figure 1: Concatenation of "proper" straight lines
$\mathrm{A} \stackrel{\mathrm{r}}{\longrightarrow} \mathrm{B}=\mathrm{C}$
$\mathrm{C} \stackrel{\mathrm{S}}{\longrightarrow} \mathrm{D}$
$\mathrm{A} \longrightarrow \mathrm{r} ; \mathrm{s}$

Figure 2: Concatenations with single-point straight lines
$\mathrm{A} \stackrel{\mathrm{r}}{\longrightarrow} \mathrm{B}=\mathrm{B}$
$\mathrm{A} \stackrel{\mathrm{r}}{\longrightarrow} \mathrm{B}$
$\mathrm{A}=\mathrm{A} \stackrel{\mathrm{r}}{\longrightarrow} \mathrm{B}$
$\mathrm{A} \stackrel{\mathrm{r}}{\longrightarrow} \mathrm{B}$ 
We now formulate these ideas in an abstract setting.

Consider a magnitude $t$. Call $\mathrm{t}$ left trivial iff $\mathrm{t} ; \mathrm{b} \preceq \mathrm{b}$, for some magnitude $\mathrm{b}$; call $\mathrm{t}$ right trivial iff $\mathrm{a} ; \mathrm{t} \preceq \mathrm{a}$, for some magnitude $\mathrm{a}$ (see Tab. 1, p. 159). A magnitude is trivial iff it is left or right trivial (see Tab. 2, p. 159). A magnitude is proper iff it is not trivial.

Table 1: Left and right trivial magnitudes

$\begin{array}{cc}\mathrm{t}: \text { left trivial } & \mathrm{t}: \text { right trivial } \\ \mathbb{1} & \mathbb{\Downarrow} \\ \exists \mathrm{b}(\mathrm{t} ; \mathrm{b} \preceq \mathrm{b}) & \exists \mathrm{a}(\mathrm{a} ; \mathrm{t} \preceq \mathrm{a})\end{array}$

Table 2: Trivial magnitude $\mathrm{t}$

$$
\mathrm{t} \in \operatorname{Trv} \quad \Leftrightarrow \quad[\underbrace{\exists \mathrm{b}(\mathrm{t} ; \mathrm{b} \preceq \mathrm{b})}_{\text {left trivial }} \text { or } \underbrace{\exists \mathrm{a}(\mathrm{a} ; \mathrm{t} \preceq \mathrm{a})}_{\text {right trivial }}]
$$

The next result characterizes left and right trivial magnitudes via equivalence.

Lemma 3.1 (Trivial). Magnitude $\mathrm{t}$ is left trivial iff $\mathrm{t} ; \mathrm{b} \sim \mathrm{b}$, for some magnitude $\mathrm{b}$. Magnitude $\mathrm{t}$ is right trivial iff a; $\mathrm{t} \sim \mathrm{a}$, for some magnitude a.

Proof. By the domination for ; and the definition of $\sim$ in Lemma 2.2 ( $\sim$ from $\preceq$, p. 157). ${ }^{8}$

The trivial magnitudes are closed under equivalence.

Corollary 3.1 (Trivial and $\sim$ ). If $\mathrm{c} \sim \mathrm{d}$, then $\mathrm{c}$ is trivial iff $\mathrm{d}$ is trivial.

Proof. By Lemma 3.1 (Trivial, pp. 159) and properties $(\operatorname{Rf}[\sim])(2, \mathrm{p} .154)$ and $(\operatorname{Tr}[\sim])$ (2. p. 154), and Lemma 3.3 (Properties of ; pp. 161). ${ }^{9}$

As Fig. 2 (p. 158) suggests, a trivial magnitude is "absorbed" by the result of addition. Example 3.2 (p. 159) examines cases of polygons and sides.

Example 3.2 (Trivial gluings). Fig. 3(p. 160) illustrates cases of gluing a triangle and its side: compared to the triangle, its sides have lower order. ${ }^{10}$

The preceding considerations suggest some basic properties of simple addition, which we assume associative: $\mathrm{a} ;(\mathrm{b} ; \mathrm{c})=(\mathrm{a} ; \mathrm{b}) ; \mathrm{c}$. 
Figure 3: Gluing triangle and side

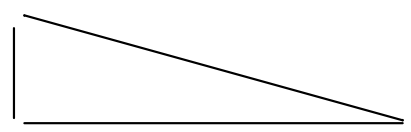

;

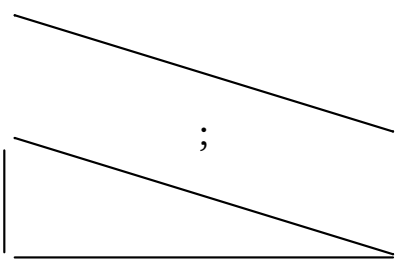

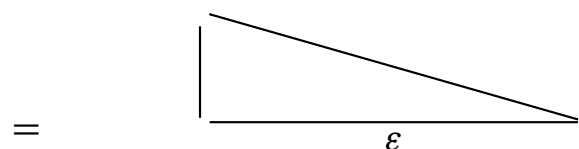

$=$

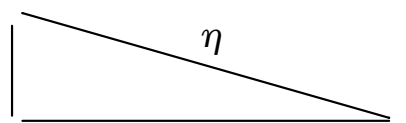

(Dm) Domination

for all $a$, $b$ : $a \preceq a$; $b$ and $b \preceq a$; $b$.

(Mn) Monotonicity

for all $a, b, c, d$ : if $a \preceq c$ and $b \preceq d$, then $a$; $b \preceq c$; .

(Cn) Non-trivial cancellations

left and right cancellations

$(\leftarrow)$ Left cancellation

for proper $b$, $c$ : if $a$; $b \preceq a$; $c$, then $b \preceq c$.

$(\rightarrow)$ Right cancellation

for proper $a$, $b$ : if $a$; $c \preceq b$; , then $a \preceq b$.

The next result gives some numerical models for simple addition ; (see also Tab. 3 . Numerical models for ; , p. 161). ${ }^{11}$

Lemma 3.2 (Models for ;). The basic properties of ; have numerical models (with $\mathrm{a} \preceq \mathrm{b}$ iff $\mathrm{a} \leq \mathrm{b}):(\mathbb{N})$ naturals with $\mathrm{a} ; \mathrm{b}:=\mathrm{a}+\mathrm{b}\left(\right.$ sum); $\left(\mathbb{N}_{1}\right)$ naturals above 1 with $\mathrm{a} ; \mathrm{b}:=\mathrm{a} \cdot \mathrm{b}$ (product); $\left(\mathbb{Q}_{0}\right)$ non-negative rationals with $\mathrm{a} ; \mathrm{b}:=\mathrm{a}+\mathrm{b}$ (sum), $\left(\mathbb{Q}_{1}\right)$ rationals above 1 with $\mathrm{a} ; \mathrm{b}:=\mathrm{a} \cdot \mathrm{b}$ (product), $\left(\mathbb{R}_{0}\right)$ non-negative reals with $\mathrm{a} ; \mathrm{b}:=\mathrm{a}+\mathrm{b}$ (sum) and $\left(\mathbb{R}_{1}\right)$ reals above 1 with $\mathrm{a} ; \mathrm{b}:=\mathrm{a} \cdot \mathrm{b}$ (product).

Proof. By properties of $\leq,+$ and $\cdot$. It suffices to check that the properties hold in $\left\langle\mathbb{R}_{0}: \leq,+\right\rangle$ and $\left\langle\mathbb{R}_{1}: \leq, \cdot\right\rangle$. Then, these universal properties will hold in the substructures $\langle\mathbb{N}: \leq,+\rangle,\left\langle\mathbb{N}_{1}: \leq, \cdot\right\rangle,\left\langle\mathbb{Q}_{0}: \leq,+\right\rangle$ and $\left\langle\mathbb{Q}_{1}: \leq, \cdot\right\rangle .^{12}$

The next result gives some derived properties of simple addition (;).

Lemma 3.3 (Properties of ;). Simple addition; has the following properties. 
Table 3: Numerical models for ;

\begin{tabular}{c|cc|cc|cc} 
Universe & $\mathbb{N}$ & $\mathbb{N}_{1}$ & $\mathbb{Q}_{0}$ & $\mathbb{Q}_{1}$ & $\mathbb{R}_{0}$ & $\mathbb{R}_{1}$ \\
\hline$;$ & $\mathrm{a}+\mathrm{b}$ & $\mathrm{a} \cdot \mathrm{b}$ & $\mathrm{a}+\mathrm{b}$ & $\mathrm{a} \cdot \mathrm{b}$ & $\mathrm{a}+\mathrm{b}$ & $\mathrm{a} \cdot \mathrm{b}$
\end{tabular}

1. Invariance for all $\mathrm{a}, \mathrm{b}, \mathrm{c}, \mathrm{d}$ : if $\mathrm{a} \sim \mathrm{c}$ and $\mathrm{b} \sim \mathrm{d}$, then $\mathrm{a} ; \mathrm{b} \sim \mathrm{c} ; \mathrm{d}$.

2. $\preceq$-cancellation $\quad$ for proper $\mathrm{b}$, $\mathrm{c}$; if $\mathrm{a} ; \mathrm{b} ; \mathrm{d} \preceq \mathrm{a} ; \mathrm{c} ; \mathrm{d}$, then $\mathrm{b} \preceq \mathrm{c}$.

3. $\sim$-cancellation for proper $\mathrm{b}, \mathrm{c}:$ if $\mathrm{a} ; \mathrm{b} ; \mathrm{d} \sim \mathrm{a} ; \mathrm{c} ; \mathrm{d}$, then $\mathrm{b} \sim \mathrm{c}$.

Proof. By Lemma 2.1 (Properties of $\sim$ and $\preceq$, p. 157), and basic properties of $\preceq$ (Sct. 2, p. 156) and of ; (Sct. 3, p. 159). ${ }^{13}$

We now examine questions concerning definability and independence. First, we examine definability of simple addition ; versus comparison $\preceq$.

Question 3.1. Can we define simple addition; from comparison $\preceq$ ?

Answer. No: we have counter-models. (See A.2, p. 166.)

Proposition 3.1 (; not definable from $\preceq$ ). Simple addition ; is not definable from comparison $\preceq$.

Proof. See A.2 (Comparison and Simple Addition: details, p. 166).

Question 3.2. Can we define comparison $\preceq$ from simple addition;?

Answer. No: we have counter-models. (See A.2, p. 167.)

Proposition 3.2 ( $\preceq$ not definable from ;). Comparison $\preceq$ is not definable from simple addition;.

Proof. See A.2 (Comparison and Simple Addition: details, p. 168).

We now examine independence of simple addition ; from comparison $\preceq$.

Question 3.3. Is each basic property of simple addition; independent from the others in the presence of those of comparison $\preceq$ ?

Answer. Yes: we have counter-models. (See A.2, p. 168,)

Proposition 3.3 (Simple addition ; independence given $\preceq$ ). Each basic property of simple addition; is independent from the others in the presence of the basic properties ( $\operatorname{Rf}[\preceq]),(\operatorname{Tr}[\preceq])$ and $(\operatorname{Tt}[\preceq])$ of comparison $\preceq$.

Proof. See A.2 (Comparison and Simple Addition: details, p. 170). 


\section{Conclusion}

We have examined magnitude equivalence $\sim$, comparison $\preceq$ and addition ; in an abstract setting. We have stressed two issues: independence and definability. For definability, we have shown a positive result and some negative results.

(+) As positive result we have: equivalence $\sim$ is definable from comparison $\preceq$, namely by $a \sim b \longleftrightarrow a \preceq b \wedge b \preceq a$.

(-) As negative results, we have the following ones.

1. Comparison $\preceq$ is not definable from equivalence $\sim$.

2. Simple addition ; is not definable from comparison $\preceq$.

3. Comparison $\preceq$ is not definable from simple addition ;.

Concerning independence, we have established some positive results about comparison $\preceq$ and simple addition ;.

( $\preceq$ ) The totality basic property ( $\operatorname{Tt}[\preceq]$ ) is independent from the other basic properties of $\preceq$, i. e. $\{\operatorname{Rf}[\preceq], \operatorname{Tr}[\preceq]\} \mid \vDash \operatorname{Tt}[\preceq]$.

(;) Each basic property of ; is independent from the others in the presence of those for $\preceq$ (see Tab. 4. Independence of basic properties of ;, p. 162).

Table 4: Independence of basic properties of ;

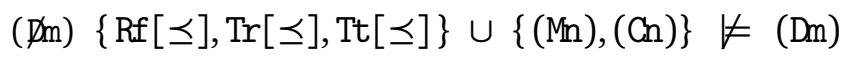

(Ch) $\{\operatorname{Rf}[\preceq], \operatorname{Tr}[\preceq], \operatorname{Tt}[\preceq]\} \cup\{(\mathrm{Dm}),(\mathrm{Nn})\} \not \models(\mathrm{Cn})$

(Y) $\{\operatorname{Rf}[\preceq], \operatorname{Tr}[\preceq], \operatorname{Tt}[\preceq]\} \cup\left\{(\operatorname{Dm}),\left(\mathrm{Cn}_{n}\right)\right\} \not \mid(\mathrm{Mn})$

Let us recall that our initial motivation was a geometrical one: to formulate and establish an abstract version of De Zolt's principle. For this purpose, one can proceed as follows:

1. Start with the basic properties of comparison $\preceq$ (cf. Sct. 2, p. 156).

2. Add equivalence $\sim$ by definition (cf. Lemma 2.2, p. 157).

3. Add the basic properties of simple addition ; (cf. 3, p. 159). 
Now, in a geometrical context, De Zolt's principle states that if a plane polygon is decomposed in any given way in a (finite) number of triangles, and then one of these triangles is removed, it is not possible to obtain a new polygonal figure equal in area to the original one. The geometrical proof of this principle is rather difficult and complex, but involves only elementary concepts.

In our abstract setting the idea of decomposition of polygons corresponds to the concept of a list of compatible proper magnitudes. A truncation of a given decomposition is then a sub-list including all its elements but one. Using these notions, we thus have the following abstract version of De Zolt's principle:

Consider a proper decomposition $\underline{p}$ of $m$. Then, for each truncation $\underline{q}$ of $\underline{p}: \underline{q}$ is not a proper decomposition of $\bar{m}$.

Our formulation of De Zolt's principle simply states that one cannot remove any proper magnitude from a proper decomposition. With the introduction of these additional concepts, one obtains what we can call a theory of "compatible" magnitudes. In this specific development of the theory of magnitudes neither the commutativity property of addition nor the Archimedean axiom are assumed.

\section{A. Some Details}

We provide here some details about comparison and equivalence (A.1. p. 163), and comparison and simple addition (A.2, p. 166).

\section{A.1. Comparison and Equivalence: details}

We provide now some details about comparison and equivalence (cf. Sct.2, pp. 156158). First, we examine models for Question 2.2 (cf. p. 157): independence of $\operatorname{Tt}[\preceq]$.

1. Finite model $\mathfrak{F}$

Consider the 3-element model with $\preceq=\{(a, a),(a, b),(b, b),(c, c)\}$ (see Fig. 4 ; Finite model $\mathfrak{F}$, p. 164$).{ }^{14}$

2. Complex model $\mathfrak{C}$

Complex numbers with $\mathrm{a}+\mathrm{b} \cdot \mathrm{i} \preceq \mathrm{c}+\mathrm{d} \cdot \mathrm{i}$ iff $\mathrm{a} \leq \mathrm{c}$ and $\mathrm{b} \leq \mathrm{d}$ (see Fig. 5; Complex model $\mathfrak{C}, \mathrm{p}$. 164).

Lemma A.1 (Finite model $\mathfrak{F}$ ). Finite model $\mathfrak{F}$ (of Fig. 4] p. 164) satisfies $\operatorname{Rf}[\preceq]$ and $\operatorname{Tr}[\preceq]$, but not $\operatorname{Tt}[\preceq]$.

Proof. Elements b and c are incomparable. 
Figure 4: Finite model $\mathfrak{F}$
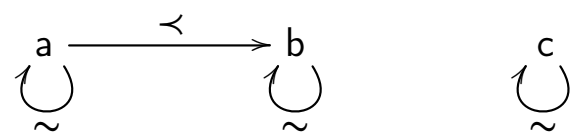

Figure 5: Complex model $\mathfrak{C}$

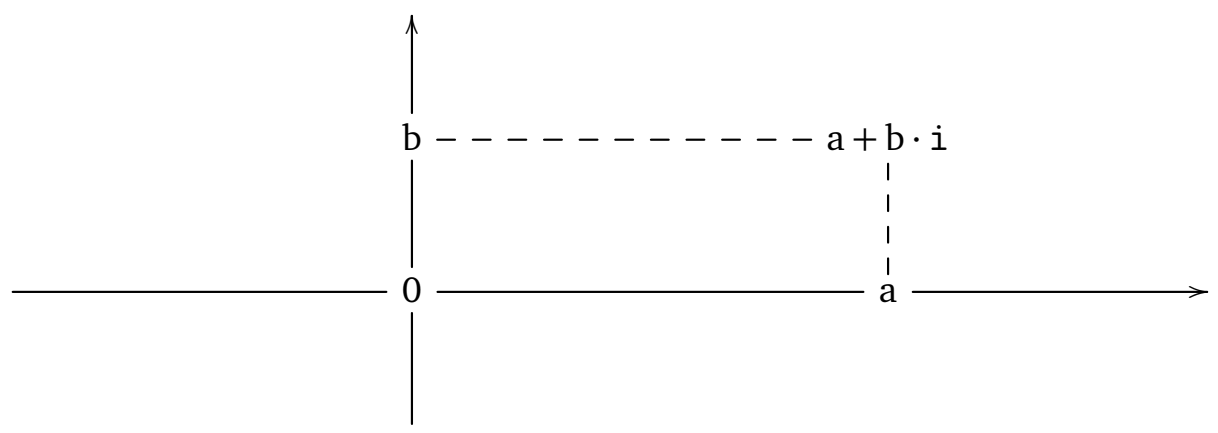

Remark A.1 (Complex model $\mathfrak{C}$ ). Complex model $\mathfrak{C}$ (of Fig. 5, p. 164) satisfies $\operatorname{Rf}[\preceq]$ and $\operatorname{Tr}[\preceq]$, but not $\operatorname{Tt}[\preceq]^{15}$

We thus have the independence of $\operatorname{Tt}[\preceq]$ from the other basic properties of $\preceq$. Proposition 2.2 (Totality independence) The totality basic property (Tt $[\preceq]$ ) is independent from the basic properties $(\operatorname{Rf}[\preceq])$ and $(\operatorname{Tr}[\preceq])$.

Proof. By Lemma A.1 (Finite model $\mathfrak{F}$, p. 163) or Remarks A.1 (Complex model $\mathfrak{C}$, p. 164).

We now examine models for Question 2.3 (cf. p. 158): definability of $\preceq$ from $\sim$.

- Finite model $\mathfrak{T}$.

Consider the 2-element model with $\preceq=\{(\perp, \perp),(\perp, T),(T, \top)\}$ (see Fig. 6 ; Finite model $\mathfrak{T}$, p. 165).

- Sine model $\mathfrak{H}$.

We can also define a model using a semicircle and sine of angle. Consider 
the points on a semicircle with center $\mathrm{X}$ and midpoint $\mathrm{A}$; define $\mathrm{U} \preceq \mathrm{V}$ iff $\sin (\widehat{\mathrm{AXU}}) \leq \sin (\widehat{\mathrm{AXV}})$ (see Fig. 7. Sine model $\mathfrak{H}, \mathrm{p} .165$ ).

Figure 6: Finite model $\mathfrak{T}$

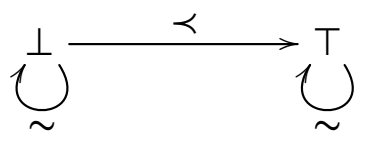

Figure 7: Sine model $\mathfrak{H}$

Lemma A.2 (Model T). In model $\mathfrak{T}$ (of Fig. 6. p. 165), the basic properties for $\sim$ and $\preceq$ hold, and the function interchanging $\perp$ and $T$ is a bijection of $\{\perp, \top\}$ that preserves $\sim$ but not $\preceq$.

Proof. See Figs. 6 (Finite model $\mathfrak{T}$ ) and 8 (Interchange in model $\mathfrak{T}$, p. 166).

Thus, comparison $\preceq$ is not definable from equivalence $\sim$.

Proposition 2.3 ( $\preceq$ not definable from $\sim$ ) Comparison $\preceq$ is not definable from equivalence $\sim$.

Proof. By Lemma A.2: Model T, p. 165 . 
Figure 8: Interchange in model $\mathfrak{T}$
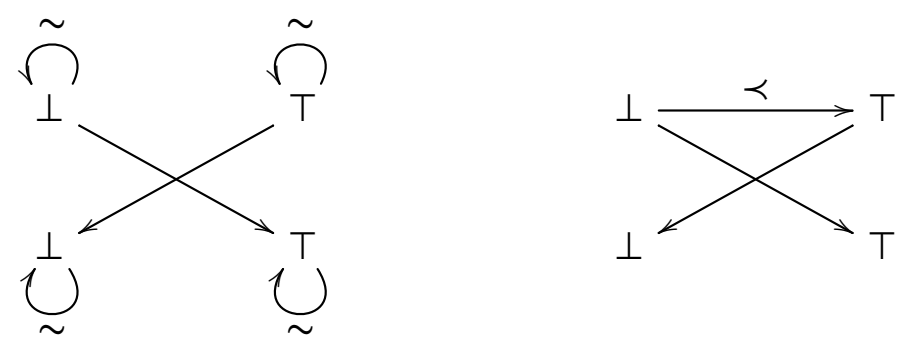

\section{A.2. Comparison and Simple Addition: details}

We now provide details about comparison and simple addition (cf. Sct. 3). We first examine counter-models for definability: ; versus $\preceq$.

Counter-model for Question 3.1 (cf. Sct. 3, p. 161): definability of ; from $\preceq$.

Model $\mathfrak{P}$ : with universe $\mathbb{Q}_{1}$ (the rationals above 1 ), $\mathrm{q} \preceq \mathrm{r}$ iff $\mathrm{q} \leq \mathrm{r}$ and $\mathrm{q} ; \mathrm{r}:=\mathrm{q} \cdot \mathrm{r}$ (product) (cf. Lemma 3.2. Models for ; pp. 160).

Lemma A.3 (Model $\mathfrak{P}$ ). In the rational model $\mathfrak{P}$ ( $c f . p$. 166), the basic properties for $\preceq$ and ; hold, and the function db assigning each $q \in \mathbb{Q}_{1}$ to its double $2 \cdot q$ is a bijection of $\mathbb{Q}_{1}$ that preserves $\preceq$ but does not preserve ;.

Proof. We have $\mathrm{q} \leq \mathrm{r}$ iff $2 \cdot \mathrm{q} \leq 2 \cdot \mathrm{r}$, and $\mathrm{db}(1 \cdot 1)=2$ whereas $\mathrm{db}(1) \cdot \mathrm{db}(1)=4$ (see also Fig. 9; Double in $\mathfrak{P}$, p. 166.

Figure 9: Double in $\mathfrak{P}$
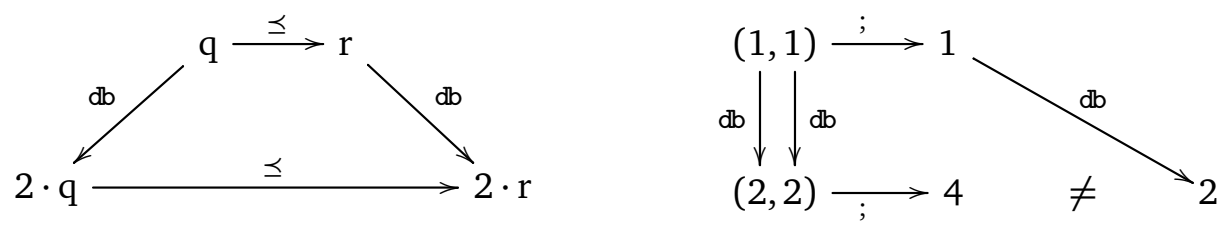

Thus, simple addition ; is not definable from comparison $\preceq$.

Proposition 3.1 (; not definable from $\preceq$ ) Simple addition ; is not definable from comparison $\preceq$. 
Proof. By Lemma A.3; Model $\mathfrak{P}, \mathrm{p} .166$.

Counter-model for Question 3.2 (cf. 3, p. 161): definability of $\preceq$ from ;.

Model $\mathfrak{S}$ : consisting of the 2 signs - and + , with $\mathrm{a} ; \mathrm{b}:=+$ (constant) and $\preceq:=\{(-,-),(-,+),(+,+)\}$ (see Fig. 10, Sign model $\mathfrak{S}$, p. 167).

Figure 10: Sign model $\mathfrak{S}$

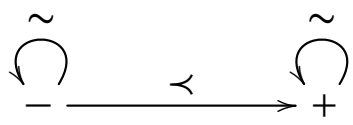

$\mathrm{a} ; \mathrm{b}:=+$ (constant)

The following results give some properties of sign model $\mathfrak{S}$.

Remark A.2 (Comparison in S). The basic properties ( $\operatorname{Rf}[\preceq]),(\operatorname{Tr}[\preceq])$ and $(\operatorname{Tt}[\preceq])$ of comparison $\preceq$ hold in model S. (See Fig. 10. Sign model S, p. 167)

Remark A.3 (Trivial in $\mathfrak{S}$ ). Signs - and + are trivial in $\mathfrak{S}^{16}$

Lemma A.4 (Non-trivial cancellation in S). Non-trivial $\preceq$-cancellation (Cn) holds in sign model $\mathfrak{S}$.

Proof. By Remark A.3 (Trivial in S, p. 167): $\operatorname{Trv}=\{-,+\}$.

Lemma A.5 (Domination in S). Domination (Dm) holds in sign model $\mathfrak{I}$.

Proof. By definition of model $\mathfrak{I}$ (cf. p. 167): $\mathrm{a} ; \mathrm{b}:=+$ and $\mathrm{a} \preceq+\succeq \mathrm{b}$.

Lemma A.6 (Monotonicity in S). Monotonicity (Mn) holds in model $\mathfrak{S}$.

Proof. By definition of ; in $\mathfrak{S}$ (cf. p. 167) and Remark A.2 (Comparison in S, p. 167): given $a, b, c$ and $d$, we always have $a ; b=+=c ; d$.

Lemma A.7 (Interchange in model $\mathfrak{S}$ ). In model $\mathfrak{S}$, the function interchanging - and + is a bijection of $\{-,+\}$ that preserves ; but does not preserve $\preceq$.

Proof. We have $\mathrm{a} ; \mathrm{b}=+$; but $-\preceq+$, whereas $+\npreceq-$ (see also Fig. 11: Sign interchange in $\mathfrak{S}, \mathrm{p} .168$.

Corollary A.1 (Model $\mathfrak{S}$ ). In model $\mathfrak{S}$ (cf. p. 167), the basic properties for $\preceq$ and ; hold, and the function interchanging - and + is a bijection of $\{-,+\}$ that preserves ; but does not preserve $\preceq$. 
Figure 11: Sign interchange in $\mathfrak{S}$

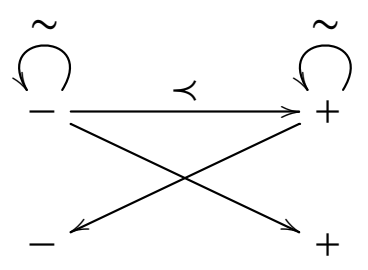

Proof. By Remark A.2 (Comparison in S, p. 167), and Lemmas A.4 (Non-trivial cancellation in S, p. 167), A.5 (Domination in S, p. 167), A.6 (Monotonicity in S, p. 167) and A.7 (Interchange in model S, p. 167).

Thus, comparison $\preceq$ is not definable from simple addition ;.

Proposition 3.2 ( $\preceq$ not definable from;) Comparison $\preceq$ is not definable from simple addition;.

Proof. By Corollary A.1: Model S, p. 167 .

We now consider models for Question 3.3 (cf. 3, p. 161): independence of each basic property ; given those of $\preceq$. We first examine counter-models for domination $(\mathrm{Dm})$ and for non-trivial cancellations $(\mathrm{Cn})$. The 2 models below use numbers with $\mathrm{a} \preceq \mathrm{b}$ iff $\mathrm{a} \leq \mathrm{b} .{ }^{17}$

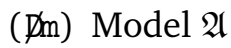
non-negative rationals $\mathbb{Q}_{0}$
$a ; b:=\frac{a+b}{2}$.
(Cn) Model $\mathfrak{M}$
naturals $\mathbb{N}$
$a ; b:=\operatorname{Max}\{a, b\}+1$.

The following results give some properties of model $\mathfrak{A}$ (cf. p. 168).

Lemma A.8 (Monotonicity in $\mathfrak{A}$ ). Monotonicity (Mn) holds in model $\mathfrak{A}$.

Proof. Monotonicity (Mn) holds because ; is an average. ${ }^{18}$

Remark A.4 (Trivial in $\mathfrak{A}$ ). Every $\mathrm{q} \in \mathbb{Q}_{0}$ is trivial in $\mathfrak{A} .{ }^{19}$

Lemma A.9 (Non-trivial cancellation in $\mathfrak{A}$ ). Non-trivial $\preceq$-cancellation (Cn) holds in model $\mathfrak{A}$.

Proof. By Remark A.4 (Trivial in $\mathfrak{A}$, p. 168): $\operatorname{Trv}=\mathbb{Q}_{0}$.

Lemma A.10 (Domination in $\mathfrak{A})$. Domination (Dm) does not hold in model $\mathfrak{A}$. 
Proof. Domination (Dm) fails because ; is an average. ${ }^{20}$

Corollary A.2 (Model $\mathfrak{A}$ ). In model $\mathfrak{A}$ (cf. p. 168), the basic properties of $\preceq$ and the ;-properties ( $\mathrm{Mn})$ and $(\mathrm{Cn})$ hold, but domination (Dm) fails.

Proof. By Lemmas A.8 Monotonicity in $\mathfrak{A}$, p. 168), A.9 (Non-trivial cancellation in $\mathfrak{A}$, p. 168) and A.10 (Domination in $\mathfrak{A}, \mathrm{p} .168$ ).

The following results give some properties of model $\mathfrak{M}$ (cf. p. 168).

Remark A.5 (Addition; in $\mathfrak{M}$ ). For all $\mathrm{a}, \mathrm{b} \in \mathbb{N}: \mathrm{a}<\operatorname{Max}\{\mathrm{a}, \mathrm{b}\}>\mathrm{b}^{21}$

Lemma A.11 (Domination in $\mathfrak{M})$. Domination (Dm) holds in model $\mathfrak{M}$.

Proof. By Remark A.5; Addition ; in $\mathfrak{M}$, p. 169 .

Lemma A.12 (Monotonicity in $\mathfrak{M})$. Monotonicity $(\mathrm{Mn})$ holds in model $\mathfrak{M}$.

Proof. By totality (Tt $[\preceq]$ ) (cf. Lemma 3.2. Models for ; pp. 160). ${ }^{22}$

Remark A.6 (Trivial in $\mathfrak{M})$. Every natural $\mathrm{n} \in \mathbb{N}$ is proper in $\mathfrak{M} .^{23}$

Lemma A.13 (Non-trivial cancellation in $\mathfrak{M})$. Non-trivial $\preceq$-cancellations $(\mathrm{Cn})$ do not hold in model $\mathfrak{E}$.

Proof. Cancellations fail because ; loses information. ${ }^{24}$

Corollary A.3 (Model $\mathfrak{M})$. In model $\mathfrak{M}$ (cf. p. 168), the basic properties of $\preceq$ and the ;-properties (Mn) and (Dm) hold, but cancellations $(\mathrm{Cn})$ fail.

Proof. By Lemmas A.11Domination in $\mathfrak{M}$, p. 169, A.12 Monotonicity in $\mathfrak{M}$, p. 169) and A.13 (Non-trivial cancellation in $\mathfrak{M}$, p. 169).

We now examine a model for the independence of ;-monotonicity. Our model is based on the extended naturals $\underline{\mathbb{N}}$ : the usual naturals with a negative zero $\underline{0}$ preceding them (see Fig. 12, Extended naturals $\underline{\mathbb{N}}$, p. 169.)

Figure 12: Extended naturals $\underline{\mathbb{N}}$

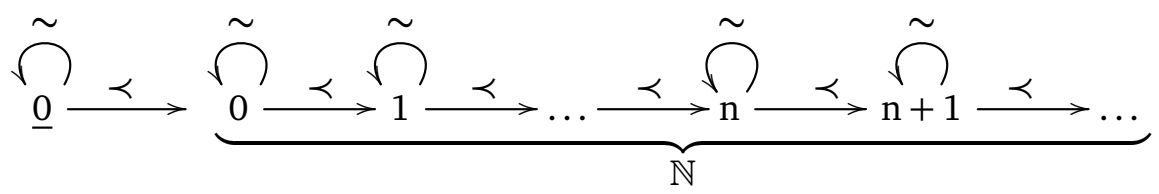


Table 5: Functions $\|$ and $\zeta$ in model $\mathfrak{E}$

$$
|\mathrm{n}|:=\left\{\begin{array}{ll}
0 & \text { if } \mathrm{n}=\underline{0} \\
\mathrm{n} & \text { if } \mathrm{n} \in \mathbb{N}
\end{array} \quad \zeta(\mathrm{n}):= \begin{cases}0 & \text { if } \mathrm{n}=0 \\
1 & \text { if } \mathrm{n} \neq 0\end{cases}\right.
$$

Model $\mathfrak{E}$ : the extended naturals $\mathbb{N}$, with $\mathrm{a} ; \mathrm{b}:=|\mathrm{a}|+|\mathrm{b}|+\zeta(\mathrm{a})$, where functions $\|$ and $\zeta$ are given in Tab. 5, p. 170 .

The following results give some properties of model $\mathfrak{E}$ (cf. p. 169).

Remark A.7 (Comparison in $\mathfrak{E})$. The basic properties ( $\operatorname{Rf}[\preceq]),(\operatorname{Tr}[\preceq])$ and $(\operatorname{Tt}[\preceq])$ of comparison $\preceq$ hold in model $\mathbb{E}$. (See Fig. 12 , Extended naturals $\mathbb{N}$, p. 169.)

Remark A.8 (Function $\|$ in $\mathfrak{E}$ ). For every $\mathrm{n} \in \underline{\mathbb{N}}:|\mathrm{n}| \succeq \mathrm{n}^{25}$

Remark A.9 (Trivial 0 in $\mathfrak{E}$ ). Natural $0 \in \underline{\mathbb{N}}$ is trivial in $\mathfrak{E}^{26}$

Remark A.10 (Monotonicity in $\mathfrak{E}$ ). Monotonicity (Mn) fails in model $\mathfrak{E}:$ take $\mathrm{a}=\underline{0}$ and $\mathrm{b}=\mathrm{c}=\mathrm{d}=0 .{ }^{27}$

Lemma A.14 (Domination in $\mathfrak{E})$. Domination (Dm) holds in model $\mathfrak{E}$.

Proof. By Remark A.8 (Function || in E, p. 170). ${ }^{28}$

Lemma A.15 (Non-trivial cancellation in $\mathfrak{E}$ ). Non-trivial $\preceq$-cancellations $(\mathrm{Cn}$ ) hold in model E.

Proof. By Remark A.9 (Trivial 0 in E, p. 170. ${ }^{29}$

Corollary A.4 (Model $\mathfrak{E}$ ). In model $\mathfrak{E}$ (cf. p. 169), the basic properties of comparison $\preceq$,;-domination $(\mathrm{Dm})$ and non-trivial $\preceq$-cancellations $(\mathrm{Cn})$ hold, but ;-monotonicity $(\mathrm{Mn})$ fails.

Proof. By Remarks A.7 (Comparison in E, p. 170) and A.9 (Trivial 0 in E, p. 170), and Lemmas A.14 Domination in E, p. 170) and A.15 (Non-trivial cancellation in $\mathfrak{E}$, p. 170).

Proposition 3.3 (Simple addition ; independence given $\preceq$ ) Each basic property of simple addition; is independent from the others in the presence of the basic properties (Rf $[\preceq]$ ), $(\operatorname{Tr}[\preceq])$ and $(\operatorname{Tt}[\preceq])$ of comparison $\preceq$.

Proof. By Corollaries A.2 (Model $\mathfrak{A}$, p. 169), A.3 Model $\mathfrak{M}$, p. 169) and A.4 (Model E, p. 170). 


\section{References}

Clifford, A. 1958. Totally Ordered Commutative Semigroups. Bulletin of the American Mathematical Society 64: 305-316.

Ehrlich, P. 2006. The Rise of non-Archimedean Mathematics and the Roots of a Misconception I: The Emergence of non-Archimedean Systems of Magnitudes. Archive for History of Exact Sciences 60: 1-121.

Giovannini, E., Lassalle Casanave, A. and Veloso, P. 2017. De la práctica euclideana a la práctica hilbertiana: las teorías del área plana. Revista Portuguesa de Filosofía 73(2): 12631294.

Hadwiger, H. 1957. Vorlesungen über Inhalt, Oberfläche und Isometrie. Heidelberg: Springer.

Hale, B. 2000. Reals by Abstraction. Philosophia Mathematica 8(3): 100-123.

Hilbert, D. 1971. Foundations of Geometry. La Salle: Open Court. Translated by L. Unger from the 10th German Edition.

Hölder, O. 1901. Die Axiome der Quantität und die Lehre vom Mass. Berichten der mathematisch-physischen Classe der Königl. Sächs. Gesellschaft der Wissenschaften zu Leipzig 53: $1-64$.

Huntington, E. 1902. A Complete Set of Postulates for the Theory of Absolute Continuous Magnitude. Transactions of the American Mathematical Society 3(2): 264-279; (3): 280284.

Łomnicki, A. 1922. O zasadzie dysjunkcyi w logistyce i matematyce. Ruch filozoficzny 6(19211922): 144-146. The title means, "On the principle of disjunction in logic and mathematic". Unpublished English translation by Andrew McFarland.

McFarland, A., McFarland, J. and Smith, J. (eds.). 2014. Alfred Tarski. Early Work in Poland - Geometry and Teaching. Berlin: Birhäuser.

Satyanarayana, M. 1979. Positively Ordered Semigroups. New York: Marcel Dekker Inc.

Stein, H. 1990. Eudoxos and Dedekind: on the ancient Greek theory of ratios and its relation to the modern mathematics. Synthese 84: 163-211.

Stolz, O. 1883. Zur Geometrie der Alten, insbesondere über ein Axiom des Archimedes. Mathematische Annalen 22: 504-519.

Stolz, O. 1885. Vorlesungen über allgemeine Arithmetik. Erster Theil: Allgemeines und Arithmetik der reellen Zahlen. Leipzig: Teubner.

Zolt, A. 1881. Principii della eguaglianza di poligoni preceduti da alcuni cenni critici sulla teoria della equivalenza geometrica. Milano: Briola.

\section{Notes}

${ }^{1}$ For historical and philosophical discussions on the standard theory of magnitude see Stein (1990), Hale (2000) and Erhlich (2006). Technical expositions can be found in Clifford (1958) and Satyanarayana (1979).

${ }^{2}$ Totality (or linearity) means that any two elements of the given set are comparable.

${ }^{3}$ It is often stressed that the fact that addition satisfies monotonicity means that the ordering is compatible with the structure of a semigroup. 
${ }^{4}$ For a historical appraisal of this work, and its relation to Tarski contributions to this subject, see McFarland, McFarland and Smith (2016).

5 The geometrical theory of equivalence investigates criteria for the equality of area of polygonal figures on the basis of its decomposition and composition in polygonal components respectively congruent. For a classical axiomatic presentation of this theory see Hilbert (1971, chapter 4). A modern presentation can be found in Hadwiger (1957).

${ }^{6}$ De Zolt's principle was originally formulated by the Italian mathematician Antonio de Zolt (1847-1926) in 1881.

${ }^{7} 1$. Given $a \sim b,(\operatorname{Sm}[\sim])$ yields $b \sim a$, whence $(\sim \Rightarrow \preceq)$ yields $b \preceq$ a. $2 .(\Rightarrow)$ Given $a \sim b$, $(\sim \Rightarrow$ ) yields $a \preceq b$, and 1 . yields $b \preceq a$. $(\Leftarrow)$ Given $a \preceq b$ \& $b \preceq a,(\preceq \Rightarrow \sim)$ yields $a \sim b$.

${ }^{8}$ We always have $t ; b \succeq b$ and $a$; $t \succeq a$. So, $t ; b \preceq b$ iff $t ; b \sim b$ and $a ; t \preceq a$ iff $a$; $\sim a$.

${ }^{9}$ For instance: if $\mathrm{c} ; \mathrm{b} \sim \mathrm{b}$ and $\mathrm{c} \sim \mathrm{d}$, then $\mathrm{d} ; \mathrm{b} \sim \mathrm{c} ; \mathrm{b} \sim \mathrm{b}$.

${ }^{10}$ One may regard sides $\varepsilon$ and $\eta$ as "infinitesimals", much as points with respect to lines (cf. Fig. 2. Concatenations with single-point straight lines, p. 158).

${ }^{11}$ We also have "geometrical" models with segments and length: $a \preceq b$ iff $\lg (a) \leq \lg (b)$ and $a ; b$ as the concatenation "a followed by b" (with $\lg (a ; b)=\lg (a)+\lg (b)$ ).

${ }^{12}\left(\mathbb{R}_{0}\right):(\mathrm{Dm}) \mathrm{a} \leq \mathrm{a}+\mathrm{b} \geq \mathrm{b}$ (as $\mathrm{a}, \mathrm{b} \geq 0$ ); (Mn) $\mathrm{a} \leq \mathrm{c} \& \mathrm{~b} \leq \mathrm{d} \Rightarrow \mathrm{a}+\mathrm{b} \leq \mathrm{c}+\mathrm{b} \leq \mathrm{c}+\mathrm{d}$; (cn) $a+b=a+c \Rightarrow b=c \& a+c=b+c \Rightarrow a=b$. $\left(\mathbb{R}_{1}\right)$ : (Dm) $a \leq a \cdot b \geq b$ (as a, b $\geq 1$ ); (cn) $a \leq c \& b \leq d \Rightarrow a \cdot b \leq c \cdot b \leq c \cdot d$; (cn) a b b $\leq a \cdot c \Rightarrow b \leq c$ (as a $>0$ ) \& $\mathrm{a} \cdot \mathrm{c} \leq \mathrm{b} \cdot \mathrm{c} \Rightarrow \mathrm{a} \leq \mathrm{b}$ (as $\mathrm{c}>0)$.

${ }^{13}$ Given $\mathrm{a} \sim \mathrm{c}$ and $\mathrm{b} \sim \mathrm{d}$, we have $\mathrm{a} \preceq \mathrm{c}, \mathrm{c} \preceq \mathrm{a}, \mathrm{b} \preceq \mathrm{d}$ and $\mathrm{d} \preceq \mathrm{b}$, thus (Dm) gives $a ; b \preceq c ; d$ and $c ; d \preceq a ; b$, whence $a ; b \sim c$; d. Given $a ; b ; d \preceq a ; c ; d$, left cancellation gives $b$; $d \preceq c$; , whence right cancellation gives $b \preceq c$. $\sim$-cancellation follows from $\preceq$ cancellation.

${ }^{14}$ We also have a 2-element model with $\preceq=\{(b, b),(c, c)\}$.

${ }^{15}$ Complex numbers $1+0 \cdot i$ and $0+1 \cdot i$ are incomparable.

${ }^{16}$ Indeed: $-;+=+$ and $+;+=+$.

${ }^{17}$ So, the basic properties of $\preceq$ hold in them (cf. Lemma 3.2. Models for ; pp. 160).

${ }^{18}$ If $\mathrm{a} \leq \mathrm{c}$ and $\mathrm{b} \leq \mathrm{d}$, then $\mathrm{a} ; \mathrm{b}=\frac{\mathrm{a}+\mathrm{b}}{2} \leq \frac{\mathrm{c}+\mathrm{d}}{2}=\mathrm{c} ; \mathrm{d}$.

${ }^{19}$ By Lemma 3.1 (Trivial, pp. 159); indeed: $\frac{\mathrm{q}+\mathrm{q}}{2}=\mathrm{q}$.

${ }^{20}$ Indeed: with $a=1$ and $b=0$. we have $a ; b=1 / 2 \nsupseteq 1=a$, and, with $a=0$ and $b=1$, we have $a ; b=1 / 2 \nsupseteq 1=b$.

${ }^{21}$ By definition of ; in $\mathfrak{M}$ (cf. p. 168): $a ; b:=\operatorname{Max}\{a, b\}+1>\operatorname{Max}\{a, b\}$ and $a \leq \operatorname{Max}\{a, b\} \geq b$.

${ }^{22}$ Given $a \leq c$ and $b \leq d$, we have 2 cases. ( $\leq$ ) If $a \leq b$, then $a ; b=b+1 \leq d+1 \leq c$; . $(\geq)$ If $a \geq b$, then $a ; b=a+1 \leq c+1 \leq c ; d$.

${ }^{23}$ By Remark A.5. Addition; in $\mathfrak{M}, \mathrm{p} .169$. $\mathrm{m}<\operatorname{Max}\{\mathrm{m}, \mathrm{n}\}>\mathrm{n}$.

${ }^{24}$ With $\mathrm{a}=\mathrm{b}=1$ and $\mathrm{c}=0$, we have $\mathrm{a} ; \mathrm{b}=2=\mathrm{a} ; \mathrm{c}$ and $\mathrm{a} ; \mathrm{c}=2=\mathrm{b} ; \mathrm{c}$, but $\mathrm{b}=1 \npreceq 0=\mathrm{c}$ and $\mathrm{a}=1 \npreceq 0=\mathrm{c}$.

${ }^{25}$ Indeed: for $\underline{0},|\underline{0}|=0 \succeq \underline{0}$, and, for $\mathrm{n} \in \mathbb{N},|\mathrm{n}|=\mathrm{n} \succeq \mathrm{n}$.

${ }^{26}$ Indeed: $0 ; 0=|0|+|0|+\zeta(0)=0+0+0=0$.

${ }^{27}$ We have: $\mathrm{a} \preceq \mathrm{c}$ and $\mathrm{b} \preceq \mathrm{b}$, but $\mathrm{a} ; \mathrm{b} \npreceq \mathrm{c}$; $\mathrm{d}$, since $\mathrm{c} ; \mathrm{d}=0 ; 0=0$ (cf. Remark A.9. Trivial 0 in $\mathfrak{E}, \mathrm{p} .170)$ and $\mathrm{a} ; \mathrm{b}=\underline{0} ; 0=|0|+|\underline{0}|+\zeta(\underline{0})=0+0+1=1$. 
${ }^{28}$ We have: $a ; b=|a|+|b|+\zeta(a) \succeq|a|+|b|$ (as $\zeta(a) \geq 0$ ), $|a|+|b| \succeq|a|$ (as $|b| \geq 0$ ) and $|a| \succeq a$; thus $a ; b \succeq a$. Similarly: $a ; b \succeq b$.

${ }^{29}(\leftarrow)$ If $b=\underline{0}$, then $b \preceq c$, so consider $b \neq \underline{0}$. If $b, c \notin \operatorname{Trv}$, then $b \neq 0$ and $c \neq 0$. . Given $|a|+|b|+\zeta(a) \leq|a|+|c|+\zeta(a)$, we have $|b| \leq|c|$, so (as $b \neq \underline{0}), b=|b| \leq|c|$, thus $b \preceq|c|$. Now, if $c \neq 0$, then $|c|=c$, whence $b \preceq|c|=c$. If $c=0$, then $|c|=|0|=0$, thus $b \preceq|c|=0$, yielding $b \in\{\underline{0}, 0\}$, which contradicts the assumptions. $(\rightarrow)$ If $a=\underline{0}$, then $a \preceq b$, so consider $a \neq \underline{0}$. If $a, b \notin \operatorname{Trv}$, then $a \neq 0$ and $b \neq 0$, so $\zeta(a)=1=\zeta(b)$, so (as $a \neq \underline{0}$ ), $a=|a| \leq|b|$, thus $a \preceq|b|$. Now, if $b \neq \underline{0}$, then $|b|=b$, whence $a \preceq|b|=b$. If $b=\underline{0}$, then $|b|=|\underline{0}|=0$, thus $\mathrm{a} \preceq|\mathrm{b}|=0$, yielding $\mathrm{a} \in\{\underline{0}, 0\}$, which contradicts the assumptions.

\section{Acknowledgments}

Previous versions of this paper have been presented at the XXII Cóloquio Conesul de Filosofía das Ciências Formais in Santa Maria (Brazil) and at the Workshop on the Theory of Magnitudes: Historical and Philosophical Perspectives, held at the Pontifical Catholic University of Rio de Janeiro (Brazil). We would like to thank the audience of these events for their helpful comments. Moreover, we are especially grateful to Edward Hermann Haeusler, Marco Panza, Luiz Carlos Pereira, Wagner de Campos Sanz and Frank Thomas Sautter for their insightful remarks and suggestions, which helped us to clarify many central ideas in this work. Finally, we are indebted to two anonymous referees of Principia, whose comments have significantly improved this paper. 